Bull. Austral. Math. Soc.

20D10, 20D20

VOL. 68 (2003) [371-378]

\title{
SOME NECESSARY AND SUFFICIENT CONDITIONS FOR $p$-NILPOTENCE OF FINITE GROUPS
}

\author{
Huaquan Wei, Yanming Wang and Xiaolei Liu
}

The purpose of this paper is to give some necessary and sufficient conditions for $p$-nilpotent groups. We extend some results, including the well-known theorems of Burnside and Frobenius as well as some very recent theorems. We also apply our results to determine the structure of some finite groups in terms of formation theory.

\section{INTRODUCTION}

A well-known theorem of Burnside [1] asserts that if some Sylow $p$-subgroup $P$ of a finite $G$ lies in the centre of its normaliser, then $G$ is $p$-nilpotent, that is, $G$ has a normal Hall $p^{\prime}$-subgroup. Another well-known result due to Frobenius [2] showed that a finite group is $p$-nilpotent if and only if the normaliser of any non-trivial $p$-subgroup is $p$ nilpotent; or the quotient group of normaliser by centraliser of any non-trivial $p$-subgroup of the group is a $p$-group.

Some authors have extended the above two theorems in different ways, for example $[3,4,5]$. In $[4]$, Ballester-Bolinches and Guo extended Burnside's theorem through the $p$-focal subgroup. They proved that a finite group $G$ is $p$-nilpotent if $P$ is a Sylow $p$ subgroup of $G$ such that $\Omega\left(P \cap G^{\prime}\right)$ is in the centre of the normaliser of $P$, where $\Omega\left(P \cap G^{\prime}\right)$ the subgroup generated by all elements $x$ of $P \cap G^{\prime}$ with $x^{p}=1$ if $p>2$ or $x^{4}=1$ if $p=2$. By using the complementation of minimal subgroups of $p$-focal subgroup, Guo and Shum in [5] also obtained a result on $p$-nilpotence of finite groups.

It is easy to show that the conditions in Ballester-Bolinches, Guo and Shum's results are not necessary. For example, let $G=\left\langle a, b \mid a^{8}=1, b^{2}=1, b^{-1} a b=a^{-1}\right\rangle$ be the dihedral group of order $2^{4}$; then $a^{6}, a^{4}$ lie in $\Omega\left(G^{\prime}\right)$ but $a^{6}$ is not in the centre of $G$ and $\left\langle a^{4}\right\rangle$ has no complement in $G$. In this paper, we shall extend the above results through some well-positioned subgroups of the $p$-focal subgroup. The conditions in our results are necessary and sufficient and hence are sharp. We also apply our results to determine the structure of some finite groups.

Received 11th March, 2003

Project supported by NSFC, NSF of Guangdong, Fund from Education Ministry of China and ARC of ZSU.

Copyright Clearance Centre, Inc. Serial-fee code: 0004-9727/03 \$A2.00+0.00. 
Recall that if $P$ is a Sylow $p$-subgroup of a finite group $G$ and $G^{\prime}$ is the commutator subgroup of $G$, the subgroup $P \cap G^{\prime}$ is called the $p$-focal subgroup of $P$ in $G$. A subgroup $H$ of a finite $G$ is said to be complemented in $G$ if there is a subgroup $K$ of $G$ such that $G=H K$ and $H \cap K=1$. A formation $\mathcal{F}$ is said to be saturated if $G / \Phi(G) \in \mathcal{F}$ implies that $G \in \mathcal{F}$.

Let $U$ be a finite $p$-group. We denote $\Omega(U)=\Omega_{1}(U)$ if $p>2$ and $\Omega(U)=\Omega_{2}(U)$ if $p=2$.

All groups considered in this paper will be finite. $G^{\mathcal{F}}$ will denote the $\mathcal{F}$-residual of $G ; \mathcal{N}, \mathcal{N}_{p}$ and $\mathcal{U}$ will denote the class of all nilpotent groups, the class of all $p$-nilpotent groups and the class of all supersolvable groups, respectively.

\section{MAin Results}

Theorem 2.1. Let $P$ be a Sylow p-subgroup of a group $G$ and let $O$ $=\left[P, O^{p}(G)\right]$, the commutator subgroup of $P$ and $O^{p}(G)$. Then the following statements are equivalent:

(1) $G$ is p-nilpotent;

(2) $\Omega(P \cap O)$ lies in the centre of $N_{G}(P)$;

(3) For any non-identity subgroup $U$ of $\Omega(P \cap O), N_{G}(U)$ is p-nilpotent;

(4) For any non-identity subgroup $U$ of $\Omega(P \cap O), N_{G}(U) / C_{G}(U)$ is a $p$-group;

(5) Every minimal subgroup of $P \cap O$ is complemented in $P$ and $N_{G}(P)$ is p-nilpotent.

Proof: $(1) \Rightarrow(5)$ : If $G$ is $p$-nilpotent, then $\left(p,\left|O^{p}(G)\right|\right)=1$, hence $P \cap O=1$ and statement (5) holds.

$(5) \Rightarrow(2)$ : Assume that (5) holds. It is clear that we may assume $P \cap O \neq 1$. Since every minimal subgroup of $P \cap O$ is complemented in $P, P \cap O$ is an elementary Abelian group. As $O=\left[P, O^{p}(G)\right] \triangleleft\left\langle P, O^{p}(G)\right\rangle=G, P \cap O \triangleleft P$. Now let $N_{1}$ be a minimal normal subgroup of $P$ contained in $P \cap O$. Then $N_{1} \leqslant Z(P)$ and $\left|N_{1}\right|=p$ by the properties of nilpotent groups. By hypothesis, there is a subgroup $P_{1}$ of $P$ such that $P=N_{1} P_{1}$ and $N_{1} \cap P_{1}=1$. Note that $P_{1} \cap O$ is still a normal subgroup of $P$. By using similar arguments, we have that $P \cap O=N_{1} \times N_{2} \times \cdots \times N_{s}$ and $N_{i} \leqslant Z(P)$. Hence $P \cap O \leqslant Z(P)$. Since $N_{G}(P)$ is p-nilpotent, $P \cap O \leqslant Z\left(N_{G}(P)\right)$, therefore statement (2) holds.

$(2) \Rightarrow(3)$ : Assume that (2) holds and (3) is false. Then $N_{G}(U)$ is not $p$-nilpotent and so $N_{G}(U)$ has a minimal non-p-nilpotent subgroup $H$ for some non-identity subgroup $U$ of $\Omega(P \cap O)$. By results of Ito [9, IV, Sata 5.4] and Schmidt [9, III, Satz 5.2], $H$ has a normal Sylow $p$-subgroup $H_{p}$ and a cyclic Sylow $q$-subgroup $H_{q}$ such that $H$ $=\left[H_{p}\right] H_{q}$. Moreover, $H_{p}$ is of exponent $p$ if $p>2$ and of exponent at most 4 if $p=2$. Since $P \leqslant N_{G}(U)$, without loss of generality, we may assume that $H_{p} \leqslant P$. On the 
other hand, the minimality of $H$ implies that $H_{p}=\left[H_{p}, H_{q}\right]=\left[H_{p}, O^{p}(H)\right]$. Hence $H_{p}$ $\leqslant P \cap\left[P, O^{p}(G)\right]=P \cap O$. It follows that $H_{p} \leqslant \Omega(P \cap O) \leqslant Z\left(N_{G}(P)\right)$. Furthermore, $N_{G}(P) \leqslant C_{G}\left(H_{p}\right)$. Denote $N=N_{G}\left(H_{p}\right)$. As $C_{G}\left(H_{p}\right) \triangleleft N_{G}\left(H_{p}\right)=N$, by the Frattini argument, $N=N_{G}\left(H_{p}\right)=C_{G}\left(H_{p}\right) N_{N}(P)$. Note that $N_{N}(P) \leqslant N_{G}(P) \leqslant C_{G}\left(H_{p}\right)$, so $N_{G}\left(H_{p}\right)=C_{G}\left(H_{p}\right)$, this implies that $H=H_{p} \times H_{q}$. This contradiction shows that statement (3) holds if (2) is true.

(3) $\Rightarrow(4)$ : Let $K$ be the normal $p$-complement of $N_{G}(U)$. Then $K U=K \times U$. Hence $K \leqslant C_{G}(U)$ and statement (4) holds.

$(4) \Rightarrow(1)$ : Assume that (4) holds and (1) is false. Then $G$ is not $p$-nilpotent and so it has a minimal non-p-nilpotent subgroup $H$. By results of Ito and Schmidt, $H$ has a normal Sylow $p$-subgroup $H_{p}$ and a cyclic Sylow $q$-subgroup $H_{q}$ such that $H=\left[H_{p}\right] H_{q}$. Moreover, $H_{p}$ is of exponent $p$ if $p$ is odd and of exponent at most 4 if $p=2$. Without loss of generality, we may assume that $H_{p} \leqslant P$. On the other hand, the minimality of $H$ implies that $H_{p}=\left[H_{p}, H_{q}\right]=\left[H_{p}, O^{p}(H)\right]$. Hence $H_{p} \leqslant P \cap\left[P, O^{p}(G)\right]=P \cap O$. It follows that $1 \neq H_{p} \leqslant \Omega(P \cap O)$. Since

$$
H / C_{H}\left(H_{p}\right)=H /\left(C_{G}\left(H_{p}\right) \cap H\right) \cong H C_{G}\left(H_{p}\right) / C_{G}\left(H_{p}\right) \leqslant N_{G}\left(H_{p}\right) / C_{G}\left(H_{p}\right),
$$

$H / C_{H}\left(H_{p}\right)$ is a $p$-group, which implies that $O^{p}(H) \leqslant C_{H}\left(H_{p}\right)$. Note that $O^{p}(H)=H$, hence $C_{H}\left(H_{p}\right)=H$ and so $H=H_{p} \times H_{q}$. This is a contradiction.

The proof of Theorem 2.1 is now complete.

The Sylow $p$-subgroup $P \cap O$ of $O$ is in fact quite familiar to us and the properties of $O$ are essential to $p$-nilpotency of a group. We state the following theorem:

THEOREM 2.2. Let $P$ be a Sylow p-subgroup of a group $G$ and let $O$ $=\left[P, O^{p}(G)\right]$, the commutator subgroup of $P$ and $O^{p}(G)$. Then

(1) $G / O$ is p-nilpotent;

(2) $P \cap O=P \cap O^{p}(G)=P \cap G^{\mathcal{N}}=P \cap G^{\mathcal{N}_{p}}$.

Proof: (1) First we have the fact that $O^{p}(G / N)=O^{p}(G) N / N$ for any normal subgroup $N$ of $G$. In fact, it is easy to see that $O^{p}(G) N / N \leqslant O^{p}(G / N)$ and we have that $(G / N) /\left(O^{p}(G) N / N\right) \cong G / O^{p}(G) N$ is a $p$-group, this implies that $O^{p}(G / N)$ $\leqslant O^{p}(G) N / N$. The above equality implies that $\left[P O / O, O^{p}(G / O)\right]=\left[P O / O, O^{p}(G) / O\right]$ $=\left[P, O^{p}(G)\right] O / O=1$. By Theorem 2.1, G/O is $p$-nilpotent.

(2) If $N$ is a normal subgroup of $G$ such that $G / N$ is $p$-nilpotent, then

$$
P N / N \cap O^{p}(G / N)=P N / N \cap O^{p}(G) N / N=\left(P \cap O^{p}(G) N\right) N / N=1,
$$

hence $P \cap O^{p}(G) N \leqslant N$. This immediately implies that $P \cap N=P \cap O^{p}(G) N$. Note that $O, G^{\mathcal{N}}$ and $G^{\mathcal{N}_{p}}$ are all contained in $O^{p}(G)$ and so (2) holds.

The following Corollary 2.3 shows that whether a group $G$ is $p$-nilpotent can be determined from the properties of the minimal subgroups of the Sylow $p$-subgroups of 
the $p$-nilpotent residual of $G$. Actually our result is a direct consequence of Theorem 2.1 and Theorem 2.2.

Corollary 2.3. Let $P$ be a Sylow $p$-subgroup of a group $G$. Then the following are equivalent:

(1) $G$ is p-nilpotent;

(2) $\Omega\left(P \cap G^{\mathcal{N}_{p}}\right)$ lies in the centre of $N_{G}(P)$;

(3) For any non-identity subgroup $U$ of $\Omega\left(P \cap G^{\mathcal{N}_{p}}\right), N_{G}(U)$ is p-nilpotent;

(4) For any non-identity subgroup $U$ of $\Omega\left(P \cap G^{\mathcal{N}_{p}}\right), N_{G}(U) / C_{G}(U)$ is a $p$ group;

(5) Every minimal subgroup of $P \cap G^{\mathcal{N}_{p}}$ is complemented in $P$ and $N_{G}(P)$ is $p$-nilpotent.

In particular, if one of the following conditions holds, then $G$ is p-nilpotent:

(i) $\Omega\left(P \cap G^{\prime}\right)$ is contained in the centre of $N_{G}(P)$ ([4, Theorem 1]);

(ii) Every minimal subgroup of $P \cap G^{\prime}$ is complemented in $N_{G}(P)$ and $N_{G}(P)$ is p-nilpotent ([5, Theorem 2.1]).

Proof: By Theorem 2.2, the equivalent conditions are direct results. Note that $G / N$ is Abelian $\Rightarrow G / N$ is nilpotent $\Rightarrow G / N$ is $p$-nilpotent. We have that $G^{\prime} \leqslant G^{\mathcal{N}}$ $\leqslant G^{\mathcal{N}_{p}}$. (2) implies (i), (5) implies (ii).

Furthermore, if $p$ is the smallest prime divisor of $|G|$, then the assumption that $N_{G}(P)$ is $p$-nilpotent in Theorem 2.1 (4) (or Theorem $2.3(4)$ ) can be removed.

THEOREM 2.4. Let $p$ be the smallest prime divisor of the order of a finite group $G$ and $P$ be a Sylow p-subgroup of the group $G$. Then $G$ is p-nilpotent if and only if every minimal subgroup of $P \cap O$ is complemented in $N_{G}(P)$, where $O=\left[P, O^{p}(G)\right]$.

'Proof: We only need to prove the 'if' part.

Write $N=N_{G}(P)$ and assume that $N<G$. Since $P \cap\left[P, O^{p}(N)\right] \leqslant P \cap O$, every minimal subgroup of $P \cap\left[P, O^{p}(N)\right]$ is complemented in $N_{N}(P)=N_{G}(P)$. We may using induction to assume that $N$ is $p$-nilpotent since $N$ satisfies all the hypothesis of $G$. This implies that $G$ is $p$-nilpotent by [6, Lemma $1(1)]$ and Theorem 2.1.

Now we consider the case where $N_{G}(P)=G$. If further $P \cap O=O \neq 1$, then we can take an element $x$ in $O$ of order $p$. By hypothesis, there is a subgroup $M$ such that $G=\langle x\rangle M$ and $\langle x\rangle \cap M=1$. Since $|G: M|=p$ and $p$ the smallest prime divisor of $|G|$, we see that $M$ is a normal subgroup of $G$. Now $G / M \cong\langle x\rangle$ is cyclic, so $\langle x\rangle \leqslant O \leqslant G^{\prime} \leqslant M$, a contradiction. Hence $O=\left[P, O^{p}(G)\right]=1$ and so $G$ is p-nilpotent by Theorem 2.1.

This completes the proof of Theorem 2.4 .

The following Corollary 2.5 is a direct result of Theorem 2.4.

COROllary 2.5. ([5, Theorem 2.2].) Let $p$ be the smallest prime divisor of $|G|$ and $P$ be a Sylow p-subgroup of the group $G$. Then $G$ is p-nilpotent if every minimal subgroup of the $p$-focal subgroup $P \cap G^{\prime}$ is complemented in $N_{G}(P)$. 
REMARK 2.6. The assumption that every minimal subgroup of $P \cap O$ is complemented in $N_{G}(P)$ in. Theorem 2.4 can not be replaced by every minimal subgroup of $P \cap O$ is complemented in $P$. For example, let $P$ be the Sylow 2-subgroup of $A_{4}$, the alternating group of degree 4; then every minimal subgroup of $P \cap O=P$ is complemented in $P$, but $A_{4}$ is not 2-nilpotent.

In the case $p=2$, the following theorem generalises $[4$, Theorem 2] and the proof of our result is better than that in [4, Theorem 2].

THEOREM 2.7. Let $P$ be a Sylow 2-subgroup of a group $G$ and assume that $P$ is quaternion-free. Then $G$ is 2-nilpotent if and only if $\Omega_{1}(P \cap O)$ is contained in $Z(P)$ and $N_{G}(P)$ is 2-nilpotent, where $O=\left[P, O^{2}(G)\right]$.

Proof: It is sufficient to prove the 'if' part.

Assume it is false and let $G$ be a counter-example of minimal order. Then $G$ has a minimal non-2-nilpotent subgroup $H$. According to results of Ito and Schmidt, $H$ has a normal Sylow 2-subgroup $H_{2}$ and a cyclic Sylow $q$-subgroup $H_{q}$ such that $H=\left[H_{2}\right] H_{q}$, where $q \neq 2$. Moreover, by the minimality of $H, H_{2}=\left[H_{2}, H_{q}\right]=\left[H_{2}, O^{2}(H)\right]$, so $H_{2}$ can be considered as a subgroup of $P \cap O$. Now, $1 \neq \Omega_{1}\left(H_{2}\right) \leqslant \Omega_{1}(P \cap O) \leqslant Z(P)$. Hence $P$ is contained in $C_{G}\left(\Omega_{1}\left(H_{2}\right)\right)$, which is a normal subgroup of $N=N_{G}\left(\Omega_{1}\left(H_{2}\right)\right)$. By the Frattini argument, $N=N_{G}\left(\Omega_{1}\left(H_{2}\right)\right)=C_{G}\left(\Omega_{1}\left(H_{2}\right)\right) N_{N}(P)$. Since $N_{N}(P) \leqslant N_{G}(P)$ and $N_{G}(P)$ is 2-nilpotent, $N_{N}(P)$ is 2-nilpotent. Let $K$ be a 2-complement of $N_{N}(P)$. Then $K$ centralises $P$ and so does $\Omega_{1}\left(H_{2}\right)$. Thus $P$ and $K$ are both contained in $C_{G}\left(\Omega_{1}\left(H_{2}\right)\right)$. Therefore $N=N_{G}\left(\Omega_{1}\left(H_{2}\right)\right)=C_{G}\left(\Omega_{1}\left(H_{2}\right)\right)$. If $N$ is a proper subgroup of $G$, then by the minimality of $G, N$ is 2-nilpotent as $N$ satisfies the hypothesis of the theorem. But $H$ lies in $N$, so $H$ is 2-nilpotent, a contradiction. Consequently, $N=G$, that is, $\Omega_{1}\left(H_{2}\right) \leqslant Z(G)$. Hence $\Omega_{1}\left(H_{2}\right) \leqslant Z(H)$. On the other hand, $H^{\mathcal{N}} \neq 1$, otherwise $H$ is nilpotent, also a contradiction. Note that $H / H_{2}$ is nilpotent, so $H^{\mathcal{N}} \leqslant H_{2}$. Now we have that $1 \neq \Omega_{1}\left(H^{\mathcal{N}}\right) \leqslant H_{2} \cap H^{\mathcal{N}} \cap Z(H)$, this contrary to a result of Dornhoff ([8, Theorem 2.8]). Therefore $H_{2} \cap H^{\mathcal{N}} \cap Z(H)=1$.

This completes our proof.

Corollary 2.8. ([4, Theorem 2].) Let $P$ be a Sylow 2-subgroup of a group $G$ and assume that $P$ is quaternion-free. If $\Omega_{1}\left(P \cap G^{\prime}\right)$ is contained in $Z(P)$ and $N_{G}(P)$ is 2-nilpotent, then $G$ is 2-nilpotent.

\section{Applications}

LEMma 3.1. ([7, Theorem 3.1].) Let $\mathcal{F}$ be a saturated formation containing $\mathcal{U}$, the class of supersolvable groups. Let $H$ be a normal subgroup of a group $G$ such that $G / H \in \mathcal{F}$. If for any maximal subgroup $M$ of $G$, either $F(H) \leqslant M$ or $F(H) \cap M$ is a maximal subgroup of $F(H)$, then $G$ lies in $\mathcal{F}$.

THEOREM 3.2. Let $\mathcal{F}$ be a saturated formation containing $\mathcal{N}$, the class of nilpo- 
tent groups. Let $H$ be a normal subgroup of a group $G$ such that $G / H \in \mathcal{F}$. Let $O=\left[P, O^{p}(G)\right]$. If $\Omega(P \cap O) \leqslant Z\left(N_{G}(P)\right)$ for any prime divisor $p$ of the order of $H$ and any Sylow p-subgroup $P$ of $H$, then $G$ lies in $\mathcal{F}$.

Proof: Suppose that the theorem is false and let $G$ be a counter-example of minimal order. Let $p$ be a prime divisor of $|H|$. Since $\left[P, O^{p}(H)\right] \leqslant O$, we have that $\Omega\left(P \cap\left[P, O^{p}(H)\right]\right) \leqslant \Omega(P \cap O) \leqslant Z\left(N_{G}(P)\right)$. Note that $N_{H}(P) \leqslant N_{G}(P)$, so $\Omega\left(P \cap\left[P, O^{p}(H)\right]\right) \leqslant Z\left(N_{H}(P)\right)$. By applying Theorem 2.1, we know that $H$ is $p$ nilpotent. The choice of $p$ implies that $H$ is nilpotent. Now let $q$ be a prime divisor of $|H|$ different from $p$ and $Q$ a Sylow $q$-subgroup of $H$. Then $Q$ is a characteristic subgroup of $H$ and hence it is a normal subgroup of $G$. Let $\bar{G}=G / Q$ and $\bar{H}=H / Q$. We shall show in the following that $\bar{G}$ satisfies the hypothesis of the theorem.

It is clear that $\bar{G} / \bar{H} \cong G / H \in \mathcal{F}$. For any Sylow $p$-subgroup $\bar{P}=P Q / Q$ of $\bar{H}$, we have that $N_{\bar{G}}(\bar{P})=N_{G}(P) Q / Q$, where $P \in \operatorname{Syl}_{p}(H)$ and $p \neq q$. Again, $\bar{P} \cap \bar{O}=$ $(P \cap O) Q / Q$. In fact, $O^{p}(G / Q)=O^{p}(G) / Q$, hence

$$
\bar{O}=\left[\bar{P}, O^{p}(\bar{G})\right]=\left[P Q / Q, O^{p}(G) / Q\right]=\left[P, O^{p}(G)\right] Q / Q=O Q / Q .
$$

Furthermore,

$$
\bar{P} \cap \bar{O}=P Q / Q \cap O Q / Q=(P \cap O Q) Q / Q=\left(P \cap \cap^{\prime}\right) Q / Q
$$

It implies that

$$
\Omega(\bar{P} \cap \bar{O})=\Omega(P \cap O) Q / Q \leqslant Z\left(N_{G}(P)\right) Q / Q \leqslant Z\left(N_{G}(P) Q / Q\right)=Z\left(N_{\bar{G}}(\bar{P})\right) .
$$

This implies that $\bar{G}=G / Q$ satisfies the hypothesis of the theorem.

By the minimality of $G$, we have that $G / Q \in \mathcal{F}$. If $Q \leqslant \Phi(G)$, then $G \in \mathcal{F}$ as $\mathcal{F}$ is a saturated formation, contrary to the choice of $G$. Therefore $Q \nsubseteq \Phi(G)$ and hence there is a maximal subgroup $M$ of $G$ such that $G=Q M$. Let $M_{q}$ be a Sylow $q$-subgroup of $M$. Then $G_{q}=Q M_{q}$ is a Sylow $q$-subgroup of $G$. Now let $G_{0}$ be a maximal subgroup of $G_{q}$ containing $M_{q}$. Then $G_{0}=M_{q}\left(G_{0} \cap Q\right)$ and denote $Q_{1}=G_{0} \cap Q$. Then $G_{0}=M_{q} Q_{1}$ and $Q_{1} \cap M=Q \cap M$. On the other hand, for any Sylow $r$-subgroup $M_{r}$ of $M$ with $r \neq q$, since

$$
\Omega\left(\left[Q, O^{q}\left(Q M_{r}\right)\right]\right) \leqslant \Omega\left(\left[Q, O^{q}(G)\right]\right) \cap Q M_{r} \leqslant Z(G) \cap Q M_{r} \leqslant Z\left(Q M_{r}\right),
$$

$Q M_{r}$ is $p$-nilpotent by Theorem 2.1 and so $Q M_{r}=Q \times M_{r}$. This means that $Q_{1} M_{r}$ is a group and so is $Q_{1}\left\langle M_{q}, M_{r} \mid M_{r} \in \operatorname{Syl}_{r}(M), r \neq q\right\rangle=Q_{1} M$. Note that $Q_{1} \cap M=Q \cap M$. We have that $Q_{1} M<G$ and so that $Q_{1} M=M$ by the maximality of $M$ in $G$. Therefore $M_{q}=M_{q} Q_{1}=G_{0}$ is a maximal subgroup of $G_{q}=Q M_{q}$; in particular, $Q$ normalises $M_{q}$. This implies that $Q$ normalises $M$ and so $M$ is a normal subgroup of $G$. Hence $G / M \cong Q /(Q \cap M) \in \mathcal{N} \subseteq \mathcal{F}$. Consequently, $G /(Q \cap M) \in \mathcal{F}$. Clearly, $Q \cap M<Q$. 
With the same argument, we know that there exists a normal subgroup $Q_{0}$ of $G$ contained in $Q \cap \Phi(G)$ such that $G / Q_{0} \in \mathcal{F}$. Thus we have $G \in \mathcal{F}$, a final contradiction.

The proof of Theorem 3.2 is complete.

THEOREM 3.3. Let $\mathcal{F}$ be a saturated formation containing $\mathcal{U}$, the class of supersolvable groups. Let $H$ be a normal subgroup of a group $G$ such that $G / H \in \mathcal{F}$. If for any prime divisor $p$ of $|H|$ and any Sylow $p$-subgroup $P$ of $H$, every minimal subgroup of $P \cap O$ is complemented in $N_{G}(P)$, then $G$ lies in $\mathcal{F}$, where $O=\left[P, O^{p}(G)\right]$.

Proof: Suppose that the theorem is false and let $G$ be a counter-example of minimal order. Since $\left[P, O^{p}(H)\right] \leqslant O$ and $N_{H}(P) \leqslant N_{G}(P)$, by [6, Lemma 1(1)], every minimal subgroup of $P \cap\left[P, O^{p}(H)\right]$ is complemented in $N_{H}(P)$. By applying Theorem 2.4, we know that $H$ has a Sylow tower of supersolvable type. Now let $q$ be the maximal prime divisor of $|H|$ and $Q$ a Sylow $q$-subgroup of $H$. Then $Q$ is a normal subgroup of $G$. Let $\bar{G}=G / Q$ and $\bar{H}=H / Q$. Then $\bar{G}$ satisfies the hypothesis of the theorem.

Clearly, $\bar{G} / \bar{H} \in \mathcal{F}$. For any Sylow p-subgroup $\bar{P}=P Q / Q$ of $\bar{H}, N_{\bar{G}}(\bar{P})$ $=N_{G}(P) Q / Q$ and $\bar{P} \cap \bar{O}=(P \cap O) Q / Q$, where $P \in \operatorname{Syl}_{p}(H)$ and $p \neq q$. Now for every element $\bar{x}$ of order $p$ in $\bar{P} \cap \bar{O}$, we have that $\bar{x}=x Q$ for some element $x \in P \cap O$. By hypothesis, there is a subgroup $K$ of $N_{G}(P)$ such that $N_{G}(P)=\langle x\rangle K$ and $\langle x\rangle \cap K=1$. Then $N_{\bar{G}}(\bar{P})=\overline{\langle x\rangle \bar{K}}$. If $\langle x\rangle \cap K Q \neq 1$, then $\langle x\rangle \leqslant K Q$ and so $N_{G}(P) Q=K Q$. Consequently, $|K|_{p}=|K Q|_{p}=\left|N_{G}(P) Q\right|_{p}=\left|N_{G}(P)\right|_{p}$. Thus $\langle x\rangle \leqslant P \leqslant K$, a contradiction. Hence $\langle x\rangle \cap K Q=1$ and so $\overline{\langle x\rangle} \cap \bar{K}=1$.

By the minimality of $G, G / Q \in \mathcal{F}$. For any maximal subgroup $M$ of $G$, if $Q$ is not contained in $M$, then $G=Q M$. Let $M_{q}$ be a Sylow $q$-subgroup of $M$. Then $G_{q}=Q M_{q}$ is a Sylow $q$-subgroup of $G$. Now let $G_{0}$ be a maximal subgroup of $G_{q}$ containing $M_{q}$; then $G_{0}=M_{q}\left(G_{0} \cap Q\right)$. Write $Q_{1}=G_{0} \cap Q$; then $G_{0}=M_{q} Q_{1}$ and $M_{q} \cap Q_{1}=M_{q} \cap Q$. Furthermore, $\left|Q: Q_{1}\right|=\left|M_{q} Q: M_{q} Q_{1}\right|=\left|G_{q}: G_{0}\right|=q$, that is, $Q_{1}$ is a maximal subgroup of $Q$. Now, we consider the following two cases:

(a) $\left[Q, O^{q}(G)\right]=1$.

For any prime divisor $r$ of $|M|$ with $r \neq q$ and any Sylow $r$-subgroup $M_{r}$ of $M$, $Q M_{r}=Q \times M_{r}$ because $\left[Q, M_{r}\right] \leqslant\left[Q, O^{q}(G)\right]=1$. This implies that $Q_{1} M_{r}$ is a group and so also is $Q_{1}\left\langle M_{q}, M_{r} \mid r \neq q\right\rangle=Q_{1} M$. But $Q_{1} M<G$ as $Q_{1} \cap M=Q \cap M$, so $Q_{1} M=M$, that is, $Q_{1} \leqslant M$. Therefore $Q \cap M=Q_{1}$ is a maximal subgroup of $Q$. By Lemma $3.1, G$ lies in $\mathcal{F}$, a contradiction.

(b) $\left[Q, O^{q}(G)\right] \neq 1$.

Since $Q$ and $O^{q}(G)$ are both normal in $G,\left[Q, O^{q}(G)\right]$ is also normal in $G$. Let $N$ be a minimal normal subgroup of $G$ contained in $\left[Q, O^{q}(G)\right]$ and take an element $y$ in $N$ of order $q$. Then, by the hypothesis, there is a subgroup $K$ of $G$ such that $G=\langle y\rangle K$ and $\langle y\rangle \cap K=1$. It is clear that $N \cap K$ is a normal subgroup of $G$, so $N \cap K=N$ or 1 from the minimal normality of $N$ in $G$. If $N \cap K=N$, then $\langle y\rangle \leqslant N \leqslant K$, a contradiction. Hence $N \cap K=1$ and so $N=\langle y\rangle$ is a normal subgroup of $G$ of order $q$. On the other 
hand, $K$ satisfies the hypothesis of the theorem. In fact, $K /(Q \cap K) \cong G / Q \in \mathcal{F}$. Note that $\left[Q \cap K, O^{q}(K)\right] \leqslant\left[Q, O^{q}(G)\right]$. By [6, Lemma 1.1(1)], every minimal subgroup of $\left[Q \cap K, O^{q}(K)\right]$ is complemented in $K$. Thus the minimality of $G$ implies that $K \in \mathcal{F}$. Now we have that $G / N \cong K \in \mathcal{F}$. By Lemma 3.1, $G \in \mathcal{F}$, a final contradiction.

The proof of Theorem 3.3 is now complete.

Corollary 3.4. ([5, Theorem 2.4].) Let $\mathcal{F}$ be a saturated formation containing $\mathcal{U}$, the class of supersolvable groups. And let $H$ be a normal subgroup of a group $G$ such that $G / H \in \mathcal{F}$. If for any Sylow subgroup $P$ of $H$, every minimal subgroup of $P \cap G^{\prime}$ is complemented in $N_{G}(P)$, then $G$ lies in $\mathcal{F}$.

REMARK 3.5. Theorem 3.2 and 3.3 are not true for non-saturated formation. For example, let $\mathcal{F}$ be the formation composed of all groups $G$ such that $G^{\mathfrak{U}}$, the supersolvable residual, is elementary Abelian. It is clear that $\mathcal{U} \subseteq \mathcal{F}$ and $\mathcal{F}$ is not a saturated formation. Let $G=S L(2,3)$ and $H=Z(G)$. Then $G / H \cong A_{4}$, so $G / H \in \mathcal{F}$. Other hypothesis in Theorem 3.3 are satisfied as is easy to see. But $G$ does not belong to $\mathcal{F}$.

\section{REFERENCES}

[1] W. Burnside, 'On some properties of groups of odd order', Proc. London Math. Soc. 33 (1901), 257-268.

[2] F.G. Frobenius, 'Über auflösbare Gruppen V', S.-B. Preuss Akad. Berlin (1901), 1324-1329.

[3] S. Li, 'On minimal subgroups of finite groups (III)', Comm. Algebra 26 (1998), 2453-2461.

[4] A. Ballester-Bolinches and X. Guo, 'Some results on p-nilpotence and solubility of finite groups', J. Algebra. 228 (2000), 491-496.

[5] X. Guo and K. Shum, 'The influence of minimal subgroups of focal subgroups on the structure of finite groups', J. Pure Appl. Algebra 169 (2002), 43-50.

[6] A. Ballester-Bolinches and X. Guo, 'On complemented subgroups of finite groups', Arch. Math. 72 (1999), 161-166.

[7] Y. Wang, H. Wei and Y. Li, 'A generalization of a theorem of Kramer and its applications', Bull. Austral. Math. Soc. 65 (2000), 467-475.

[8] L. Dornhoff, ' $M$-group and 2-groups', Math. $Z$. 100 (1967), 226-256.

[9] B. Huppert, Endliche Gruppen I (Springer-Verlag, Berlin, Heidelberg, New York, 1983).

School of Mathematics

Zhongshan University

Guangzhou, 510275

China

and

Deptartment of Mathematics

Guangxi Teacher's College

Nanning, 530001

China

e-mail: weihuaquan@163.com
Lingnan College and School of Math.

Zhongshan University

Guangzhou, 510275

China

email: stswym@zsu.edu.cn

Lingnan College and School of Math.

Zhongshan University

Guangzhou, 510275

China 